\title{
Targeting and silencing of rhodopsin by ectopic expression of the transcription factor KLF15
}

Salvatore Botta, ${ }^{1}$ Nicola de Prisco, ${ }^{1}$ Elena Marrocco, ${ }^{1}$ Mario Renda, ${ }^{1}$ Martina Sofia, ${ }^{1}$ Fabiola Curion, ${ }^{1}$ Maria Laura Bacci, ${ }^{2}$ Domenico Ventrella, ${ }^{2}$ Cathal Wilson, ${ }^{1}$ Carlo Gesualdo, ${ }^{3}$ Settimio Rossi, ${ }^{3}$ Francesca Simonelli, ${ }^{3}$ and Enrico Maria Surace ${ }^{1,4}$

${ }^{1}$ Telethon Institute of Genetics and Medicine, Napoli, Italy. ${ }^{2}$ Department of Veterinary Medical Sciences, University of Bologna, Bologna, Italy. ${ }^{3}$ Multidisciplinary Department of Medical, Surgical and Dental Sciences, Eye Clinic, Second University of Naples, Naples, Italy. ${ }^{4}$ Department of Translational Medicine, University of Naples Federico II, Naples, Italy.

The genome-wide activity of transcription factors (TFs) on multiple regulatory elements precludes their use as gene-specific regulators. Here we show that ectopic expression of a TF in a cellspecific context can be used to silence the expression of a specific gene as a therapeutic approach to regulate gene expression in human disease. We selected the TF Krüppel-like factor 15 (KLF15) based on its putative ability to recognize a specific DNA sequence motif present in the rhodopsin $(R H O)$ promoter and its lack of expression in terminally differentiated rod photoreceptors (the RHO-expressing cells). Adeno-associated virus (AAV) vector-mediated ectopic expression of KLF15 in rod photoreceptors of pigs enables $R$ ho silencing with limited genome-wide transcriptional perturbations. Suppression of a RHO mutant allele by KLF15 corrects the phenotype of a mouse model of retinitis pigmentosa with no observed toxicity. Cell-specific-context conditioning of TF activity may prove a novel mode for somatic gene-targeted manipulation.

Authorship note: N. de Prisco and E. Marrocco contributed equally to this work.

Conflict of interest: The authors have declared that no conflict of interest exists.

Submitted: July 27, 2017 Accepted: November 15, 2017 Published: December 21, 2017

\section{Reference information:} JCI Insight. 2017;2(24):e96560 https://doi.org/10.1172/jci. insight. 96560.

\section{Introduction}

Transcription factors (TFs) control space- and time-dependent activation or repression of genes to control biological functions (1). They regulate these genetic programs by genome-wide scanning of DNA sequences and eventually binding to discrete motifs present in gene regulatory regions (promoters and enhancers) $(2,3)$. TFs have an intrinsic ability to recognize primary nucleotide DNA sequence motifs (a base readout [see ref. 4] of typically 5-15 bp). The principles of TF protein-DNA recognition has enabled the determination of their DNA binding preferences and the design of synthetic TFs directed to specific genomic DNA sequences $(5,6)$. However, individual TFs and TF family members show differential DNA binding preferences, indicating that the TF-DNA recognition code is far from being fully elucidated (7), particularly in vivo. Local and distal chromosomal features, protein-protein interactions, and nuclear topography are emerging as determinants conditioning the DNA accessibility, binding, and ultimately activity of TFs (8-10). These features are inherent to cell-specific composition and may be envisaged as extrinsic cofactors that complement the intrinsic TF recognition properties for DNA base readout. Somatic cells of an individual organism have the same DNA sequence (syngeneic) while expressing cell-specific factors. Thus, they represent an ideal model system to study the impact of the intrinsic and the extrinsic properties on TF activity. Based on this assumption, we hypothesized that the extrinsic cell-specific context might direct the genome-wide activity of an ectopically expressed TF to a single gene target and that this approach might be used to treat a blinding disease, retinitis pigmentosa.

\section{Results}

To treat autosomal dominant retinitis pigmentosa (adRP) (11) associated with gain-of-function rhodopsin $(R H O)$ alleles, we showed recently that adeno-associated virus (AAV) vector-mediated in vivo delivery to the retina of synthetic TFs designed to bind to a 20-bp DNA sequence motif (ZF6-cis sequence) in the RHO promoter enables effective $R H O$ transcriptional repression $(12,13)$. This indicated that the DNA sequence motif is accessible for binding and is functionally relevant. Furthermore, detailed epigenomic mapping confirmed 
A

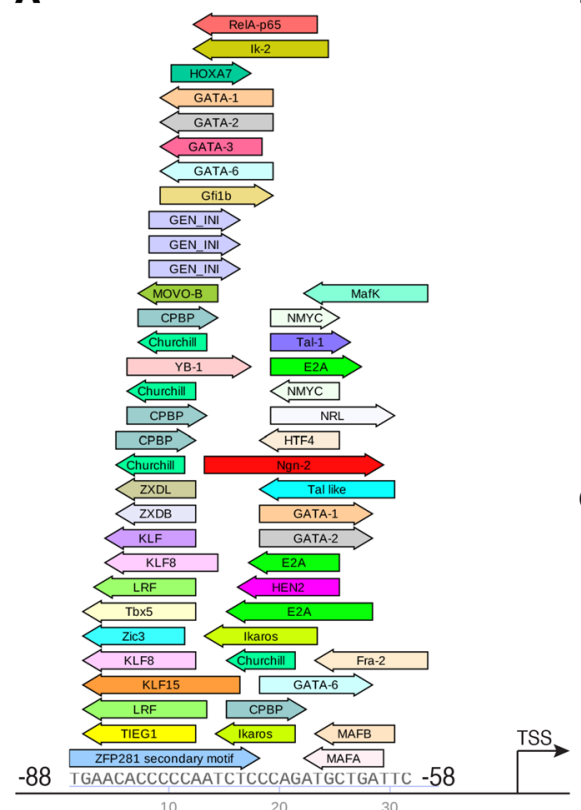

human RHODOPSIN promoter
B

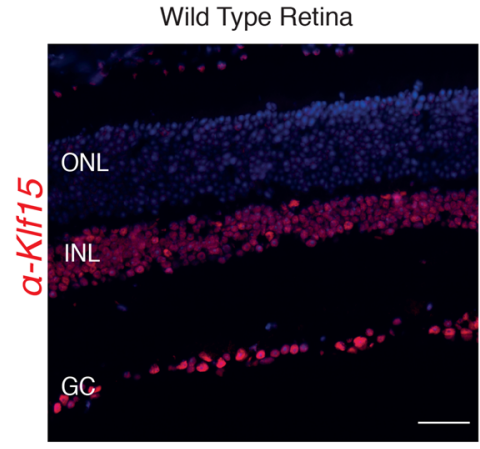

C

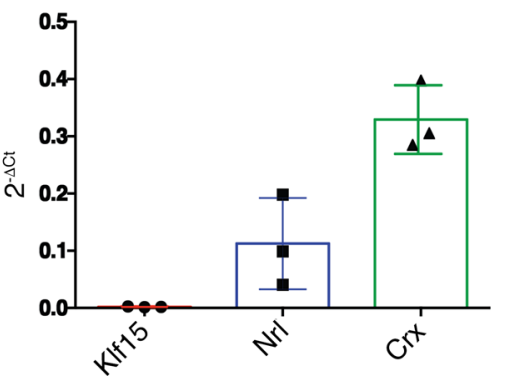

D

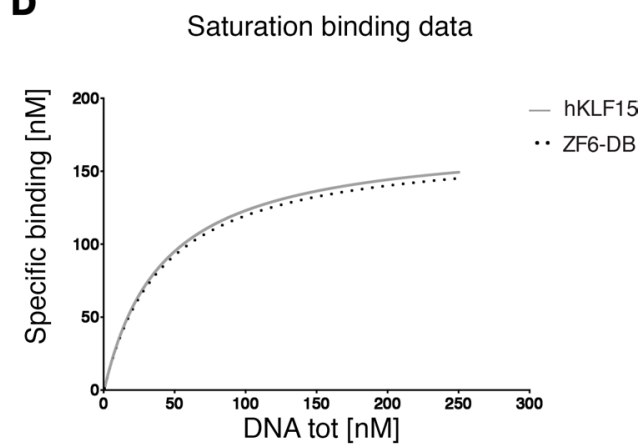

E

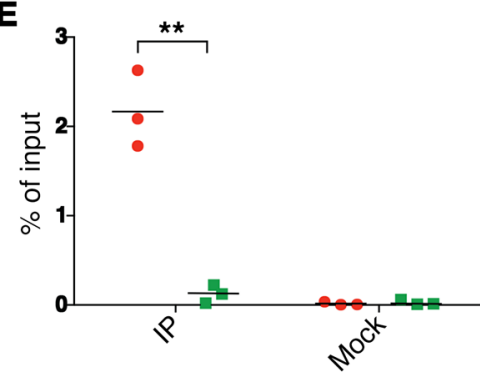

- hKLF15

eGFP

Figure 1. KLF15 is not expressed in rods and binds the human rhodopsin promoter. (A) TRANSFAC analysis of the human rhodopsin promoter identifies transcription factors (TFs) predicted to bind the rhodopsin regulatory motif $h R H O$-cis ( -88 to -58 from the transcription start site, TSS; Figure $2 \mathrm{~A}$ and refs. 12,13 ) including the TF KLF15 (orange arrow, minus strand). (B) Immunofluorescence analysis of KIf15 in C57BL6/J retina shows its absence in photoreceptors in the outer nuclear layer (ONL) and expression in the inner nuclear layer (INL) and in the ganglion cell layer (GCL). Scale bar: $50 \mu \mathrm{m}$. (C) qPCR of mRNA $\left(2^{-\Delta C t}\right)$ shows that $K I f 15$ is not expressed in porcine rods. Porcine rods transduced with AAV8-hGNAT1-eGFP $\left(1 \times 10^{12}\right.$ genome copies [gc] $)$ and FACS sorted show lack of expression of KIf15. For comparison the retina-specific cone-rod homeobox (Crx) and rod-specific neural retina leucine zipper (Nrl) TFs are shown. (D) Gel mobility shift titrations of hKLF15 and artificial ZF6-DB TF with the hRHO 65-bp oligonucleotide. In the saturation-binding experiments the nanomolar concentration of specific binding data were plotted against nanomolar increasing concentration of DNA ligand. KLF15 and the synthetic TF ZF6-DB show similar binding affinity for the target sequence $(12,13)$. (E) qPCR ChIP analysis of the human rhodopsin TSS region, after the transfection of hKLF15 in HEK293 cells, shows enrichment of binding in the Rho promoter region compared with eGFP-transfected cells. Data are shown as the mean \pm SEM. ${ }^{* *} P<0.01$ by 2 -tailed Student's $t$ test. $n=3$ independent experiments.

the accessibility of this region of the Rho promoter (14). Thus, these previous results represent a reference system to determine whether somatic ectopic gene transfer of a TF might be used to silence RHO expression. We searched initially for endogenous TFs with a DNA-binding preference for the ZF6-cis sequence motif but that are not expressed in rod photoreceptors (the $R H O$-expressing cells). To retrieve TFs we used TRANSFAC analysis (15), which provides data on eukaryotic TF consensus binding sequences (based on positional weight matrices, PWMs), using as bait a 32-bp DNA sequence centered on the ZF6-cis sequence of the human $R H O$ promoter ( -88 to -58 from the $R H O$ transcriptional start site [TSS], here named $h R H O$ cis). Among the set of retrieved TFs (Figure 1A) KLF8 and -15 belong to the Krüppel-like factor (KLF) gene family (16), which possess a zinc-finger structure (KRAB-ZNF TFs) and recognize the GT-box and the core motif CACCC present in the hRHO-cis (16). In particular, KLF15 has a wide matrix sequence highly overlapping the ZF6-cis sequence (Supplemental Table 1; supplemental material available online with this article; https://doi.org/10.1172/jci.insight.96560DS1) and is expressed throughout the retina but not in photoreceptors (17) and thus can be excluded from having a regulatory function in these cells. In addition, although $K L F 15$ exerts a wide range of regulatory functions in different organs and in system homeostasis (18-20), the mouse knockout does not exhibit prominent phenotypes (21). Before proceeding with KLF15 as a candidate for somatic ectopic expression, we confirmed that KLF15 is not expressed in terminally differentiated rod photoreceptors using immunofluorescence analysis in mouse, porcine, and human retina (Figure 1B and Supplemental Figure 1). Antibody staining showed KLF15 expression in the ganglion cell layer (GCL) and inner nuclear layers (INLs) but an apparent lack of expression in the outer nuclear layer (ONL) (Figure 1B and Supplemental Figure 1). However, in the pig retina we found expression of Klf15 also in cone photoreceptors (Supplemental Figure 1C). To further confirm that Klf15 is not expressed in rods, we used a procedure to isolate a population of porcine rods for analysis. Specifically, porcine rods were labeled 
A

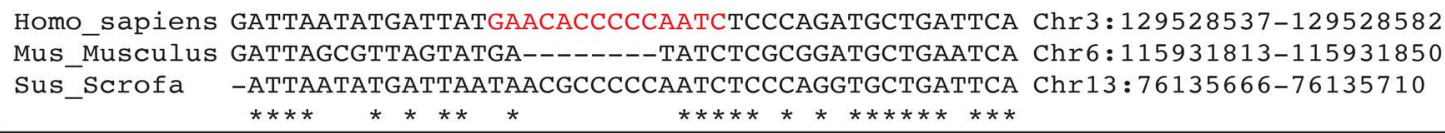

B

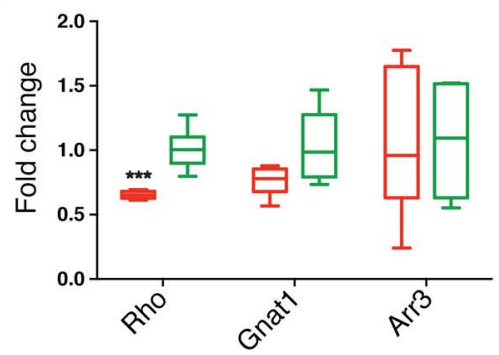

C

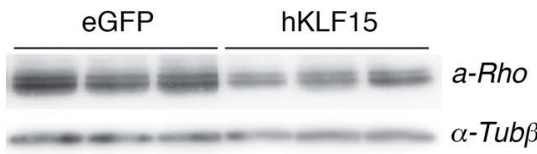

$\mathbf{F}$



D
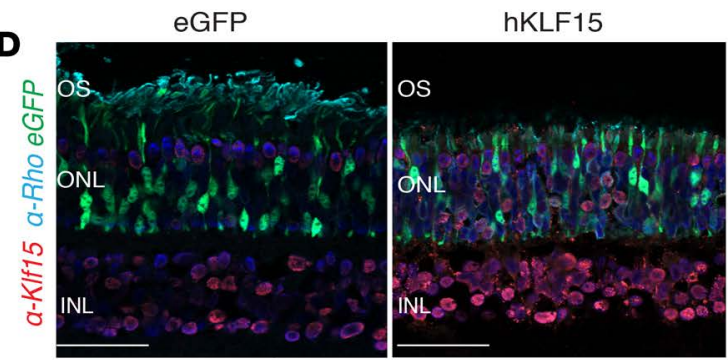

E
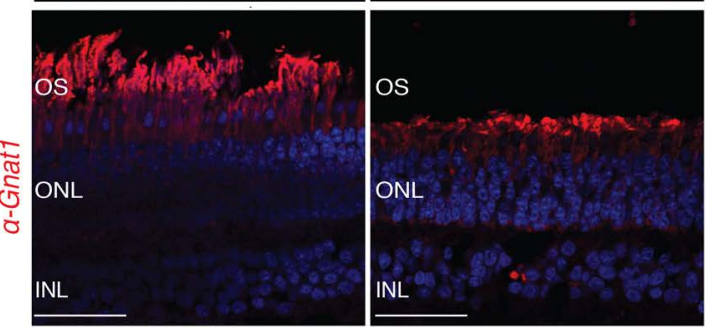

G

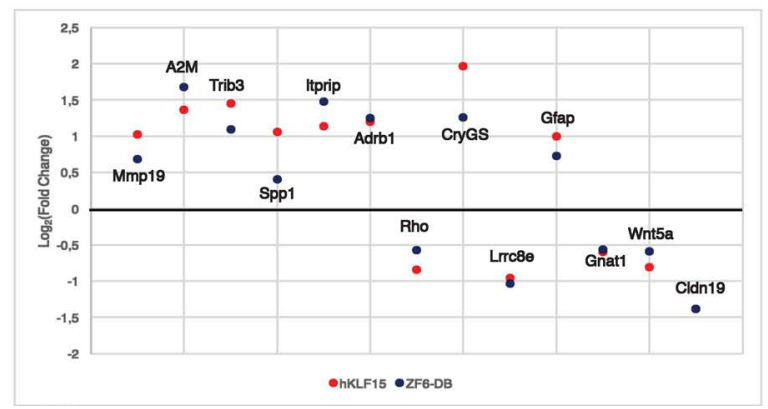

Figure 2. KLF15 ectopically expressed in porcine rod photoreceptors represses Rho expression with limited off-target effects. (A) Alignment of human, porcine, and murine rhodopsin (Rho) proximal promoter around the $h R H O$-cis. In red, the sequence recognized by KLF15 retrieved by TRANSFAC analysis (Figure 1A and Supplemental Table 1). (B) qPCR of mRNA levels (2- $\left.{ }^{-\Delta A t}\right)$ of adult porcine retina injected subretinally with AAV8-hGNAT1-hKLF15 ( $\left.n=6\right)$ or AAV8-hGNAT1-eGFP $(n=6)$ at a vector dose of $2 \times 10^{10}$ genome copies (gc) 15 days after vector delivery shows significant repression of the Rho transcript; Gnat1, guanine nucleotide-binding protein 1; Arr3, arrestin 3. Data are shown as the mean \pm SEM. ${ }^{* * *} P<0.001 ; 2$-tailed Student's $t$ test. (C) Western Blot analysis of porcine retinae injected with AAV8-hGNAT1-hKLF15 and AAV8-hGNAT1-eGFP shows the decrease in Rho protein consequent to KLF15 expression. (D) Rho (cyan) and KLF15 (red) immunofluorescence confocal analysis shows expression of hKLF15 in the outer nuclear layer (ONL) of injected retina (coinjected with AAV8-hGNAT1-eGFP, green) toward the nuclear interior of rod photoreceptor nuclei (euchromatin; see ref. 33), the collapse of the Rhodeprived outer-segment (OS), and partial retention of Rho in the cytoplasm. (E) Histological confocal immunofluorescence analysis of Gnat1 (red), which marks the soma of rods, confirmed rod-specific expression of $h K L F 15$ upon transduction with AAV8-hGNAT1-hKLF15. Scale bars: $50 \mu \mathrm{m}$. (F) Venn diagrams showing pairwise intersection of differentially expressed genes (DEGs) between $h K L F 15$ and ZF6-DB (12, 13). An adjusted $P$ value (false discovery rate < 0.1), without filtering on fold change levels, resulted in 156 and 19 DEGs, in $h K L F 15$ - and ZF6-DB-treated retinae, respectively (12, 13). (C) Transcriptional activation and repression concordances among log fold changes of the genes in common between ZF6-DB and hKLF15.

by subretinal injection of an AAV vector containing $e G F P$ under the control of the rod-specific promoter element GNAT1 (AAV8-hGNAT1-eGFP; ref. 12). Fifteen days after injection, eGFP-positive rods were dissociated and sorted by FACS and we measured Klf15 mRNA levels by quantitative real-time PCR (qPCR), but no Klf15 expression could be observed (Figure 1C). We next evaluated the affinity of human KLF15 for the hRHO-cis. KLF15 showed high affinity for the hRHO-cis, similar to that of the synthetic TF ZF6-DB (Figure 1D). Furthermore, chromatin immunoprecipitation (ChIP) showed proper hRHO-cis genomic occupancy by KLF15 (Figure 1E). These data suggest that KLF15 and the synthetic TF ZF6-DB show analogous binding properties despite protein structural differences (KLF15 has a KRAB effector domain at the N-terminus and 3 zinc fingers at the C-terminus, while ZF6-DB has 6 zinc fingers without an effector domain). 
We used the wild-type porcine retina to investigate the ability of $K L F 15$ to repress Rho expression. The hRHO-cis sequence is highly conserved between pigs and humans (Figure 2A). Subretinal injection of a low dose of an AAV8 vector containing the human $K L F 15$ ( $h K L F 15)$ under the rod-specific GNAT1 promoter in adult pigs $\left(2 \times 10^{10}\right.$ genome copies [gc] of AAV8-GNAT1-hKLF15 vector), showed that $h K L F 15$, 15 days after delivery, resulted in $45 \%$ and the $38 \%$ repression of the $R$ ho transcript and protein levels, respectively, in the transduced area (Figure 2, B and C). Consistent with these observations, morphological analysis showed the collapse of Rho-deprived outer segments (OS). Despite Rho depletion, the integrity of the ONLs was maintained at this short time point (Figure 2, D and E), in agreement with what has been observed with the synthetic TF ZF6-DB $(12,13)$. To determine genome-wide transcriptional changes that might be caused by the ectopic expression of $h K L F 15$, we evaluated retinae by RNA sequencing (RNA-Seq) 15 days after subretinal injection of an AAV8-CMV-hKLF15. In this case, the $C M V$ promoter was used to cross-compare the data sets with those generated with the synthetic TF ZF6-DB $(12,13)$. We found 156 differentially expressed genes (DEGs), of which 3 were rod-photoreceptor specific (Rho, Gnat1, and Crx, Supplemental Table 2). Thirteen of the 19 DEGs induced by the synthetic TF ZF6-DB overlapped with those of $h K L F 15$ and showed transcriptional activation and repression concordances (Figure 2, F and G). From the intersection between the 1,213 genes differentially expressed in the lung of Klf15-knockout mice (22), only 27 DEGs were shared with hKLF15 ectopic expression (data not shown).

To test whether $R H O$ repression mediated by the ectopic expression of $h K L F 15$ could produce a therapeutic effect, we delivered AAV8-GNAT1-hKLF15 into the transgenic RHO-P347S mouse model of adRP (23). This adRP mouse model harbors the P347S human RHO mutant allele, including the hRHO-cis motif, and the endogenous murine Rho alleles (23). Interestingly, despite extensive promoter conservation with humans, the murine Rho promoter diverges in the hRHO-cis sequence motif (Figure 2A). We took advantage experimentally of this sequence motif difference to determine the specificity of $h K L F 15$ for the human $h R H O$-cis RHO regulatory sequence. We expected that the selective binding and repression of the human $R H O$ transgenic promoter by $K L F 15$ would result in preservation of retinal function due to the silencing of the P347S RHO mutation. Subretinal delivery of AAV8-GNAT1-hKLF15 in P14 P347S mice resulted in significant repression of the human $R H O$ mutant transgene transcript but left unchanged expression from the endogenous murine Rho alleles (Figure 3D). The selective silencing of the P347S RHO mutation resulted in the preservation of retinal structure and function, evaluated by electroretinography (ERG) and histological analysis 30 days after delivery (Figure 3, A-C and Supplemental Figure 2). Similar human-specific P347S mutant RHO repression was observed in P14 P347S mice injected with an AAV containing the murine Klf15 orthologous gene, which shows complete conservation of the C-terminus zinc-finger DNA-binding domain and partial conservation of the N-terminus (Figure 3). Notably, these findings support the notion that the recognition of $h R H O$-cis by KLF15 is independent of the specific Rho chromosomal location (the $P 347 S$ adRP mouse model harbors the mutant $R H O$ in nonspecific loci), that local sequence features may contribute to the observed effect (24), and that the human and murine KLF15 genes based on their conservation operate similarly on the $h R H O-c i s$ sequence. To evaluate tolerability and potential toxicity of ectopic expression of Klf15 in rods, we subretinally injected adult wild-type mice with the human or the murine Klf15 gene (AAV8-GNAT1-hKLF15 or AAV8-GNAT1-mK1f15, respectively). Eighty days after delivery the retina of treated animals showed no changes in $R$ ho transcript levels (qPCR) and no detrimental effects on retinal ERG electrophysiological responses or histological appearance (Supplemental Figures 3 and 4).

\section{Discussion}

In this study we have shown that cell-specific factors, in which an ectopically expressed TF operates, restrict its activity. In particular, ectopic expression of $K L F 15$, which is involved in a wide variety of organ functions, in terminally differentiated rod photoreceptors silenced $\mathrm{RHO}$ expression with limited off-target effects. The results show that the cell-specific context may limit TF activities that control wide and coherent genetic programs, which, for instance, determine developmental and somatic photoreceptor identity transitions in the mammalian retina $(1,25,26)$. KLF15 belongs to the largest TF group (KRAB-ZNF TFs) in the mammalian genome, with an estimated repertoire of approximately $400 K R A B-Z N F$ TFs. In addition, KRAB-ZNF TFs show highly differential tissue patterns of expression $(27,28)$. Thus, in principle, this TF somatic ectopic gene transfer approach could be extended to other gene targets by combining TF preferences with cell-specific expression and genome accessibility maps $(10,14)$. Of note, gene expression profiles in diverse tissues of the human body and across individuals are being increasingly identified (29). 

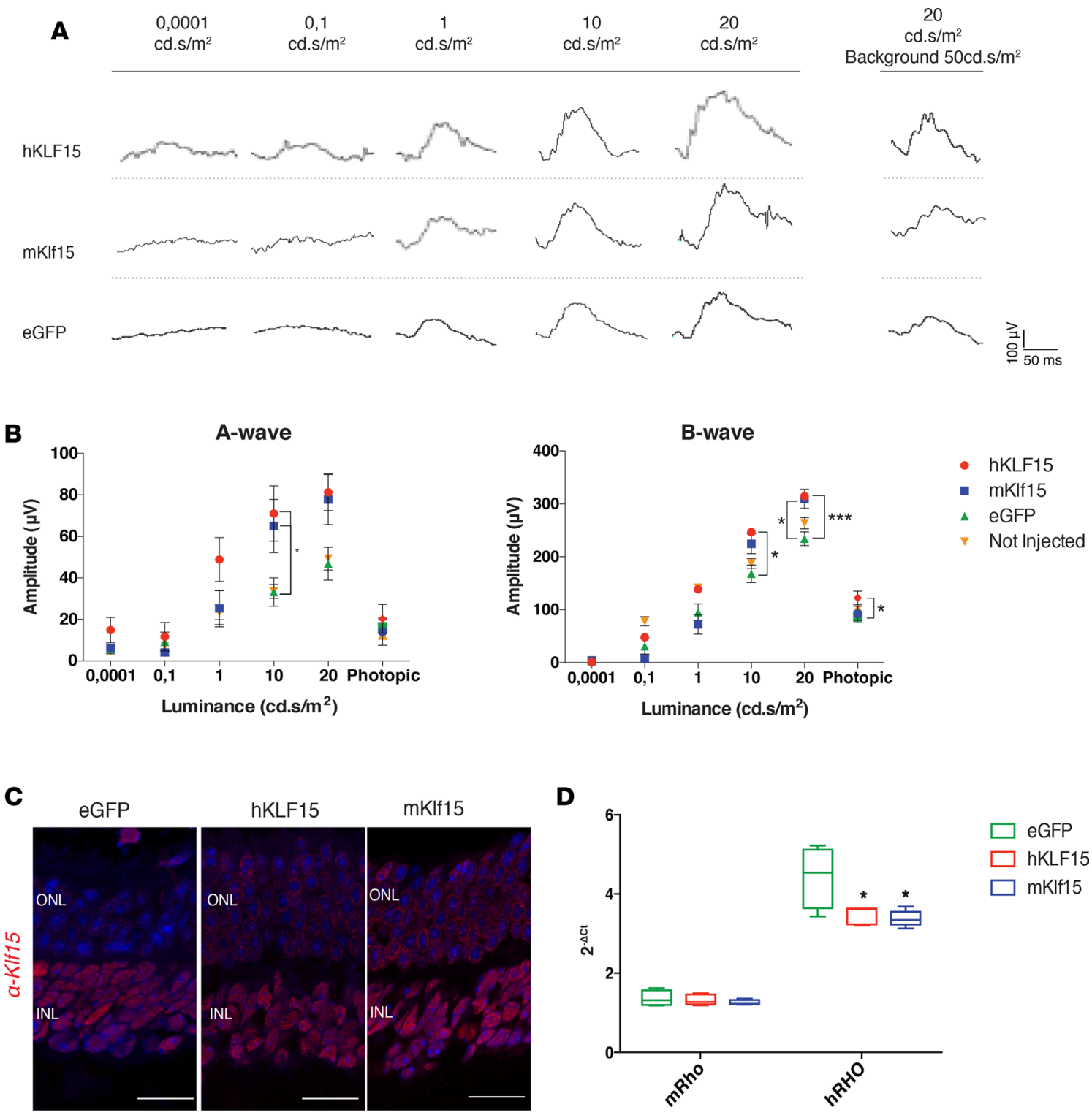

Figure 3. KLF15 ectopic expression preserves retinal function in adRP-transgenic $\mathbf{R H O}$-P3475 mice. (A) Electroretinography (ERG) traces from a representative mouse injected with AAV carrying hKLF15, mKIf15, or eGFP measured at increasing luminances (cd.s/m²). (B) ERG analysis on P347S mice subretinally injected at postnatal day 14 (P14) with AAV8-hGNAT1-hKLF15 $(n=12)$, AAV8-hGNAT1-mKIf15 $n=9$ ), AAV8-hGNAT1-eGFP ( $n=14$ ), or not injected $(n=6)$ and analyzed at P3O. Retinal responses in both scotopic (dim light) and photopic (bright light) showed that both a- and b-wave amplitudes, evoked by increasing light intensities, were more preserved in hKLF15- and mKIf15-injected eyes compared with eGFP control eyes. (C) Immunofluorescence staining of P347S mouse retina, injected at P14 with AAV8-hGNAT1-hKLF15, AAV8-hGNAT1-mKIf15, or AAV8-hGNAT1-eGFP and analyzed at P30. hKLF15- and mKIf15-treated retinae show KLF15-positive expression toward the periphery of rod photoreceptor nuclei, an inverted pattern compared with pig (Figure $2 \mathrm{D}$ and ref. 33), and higher preservation of the outer nuclear layer (ONL) compared with eGFP controls. INL, inner nuclear layer. (D) qPCR

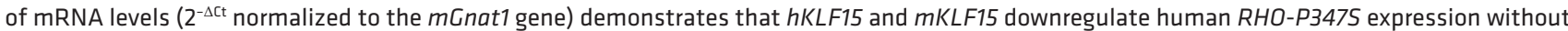
changing the endogenous wild-type murine rhodopsin transcript.

Ectopic expression of $K L F 15$ resulted in efficient Rho silencing similar to that shown by synthetic TFs $(12,13)$. Silencing of the severe $R H O-P 347 S$ gain-of-function mutation in the adRP mouse model translated into structural and functional protection of the retina from degeneration. Coupling Rho transcriptional silencing with replacement, as others and we described (30) and the safety and efficacy of AAV retinal gene transfer (31), supports further development of this strategy for the treatment of adRP. In summary, we provided a proof-of-concept of a potentially novel mode to efficiently and specifically silence a gene by ectopic expression of a TF in a cell-specific context. 


\section{Methods}

\section{Prediction of TF binding}

The promoter sequence of $R H O$ was analyzed using TRANSFAC with the "Vertebrate" database using high-quality matrices and a "Core score" and "Matrix score" higher than 0.95 . The sequence analyzed was Chr3:129528551-129528581 corresponding to -88 to -58 from the TSS of human rhodopsin.

\section{Plasmid construction}

The human KLF15 coding DNA sequence (CDS) and the murine KLF15 CDS were synthesized by Eurofins MWG. The fragments were subcloned in pAAV2.1 under the control of the CMV or $h G N A T 1$ promoter using NotI and HindIII restriction enzymes.

\section{AAV vector preparation}

AAV vectors were produced by the Telethon Institute of Genetics and Medicine (TIGEM) AAV Vector Core, by triple transfection of HEK293 cells followed by 2 rounds of $\mathrm{CsCl}_{2}$ purification. For each viral preparation, physical titers $(\mathrm{gc} / \mathrm{ml})$ were determined by averaging the titer achieved by dot-blot analysis and by PCR quantification using TaqMan (Applied Biosystems) $(12,13)$.

\section{Animal models}

All procedures were performed in accordance with institutional guidelines for animal research and all of the animal studies were approved by the authors. $P 347 \mathrm{~S}^{+/+}$animals (23) were bred in the animal facility of the Biotechnology Centre of the Cardarelli Hospital (Naples, Italy). $P 347 S^{-/}$mice were crossed with C57BL6/J mice (Charles Rivers Laboratories) to obtain the $P 347 S^{+/-}$mice.

\section{Vector administration}

Mice. Intraperitoneal injection of ketamine and medetomidine were administered $(100 \mathrm{mg} / \mathrm{kg}$ and 0.25 $\mathrm{mg} / \mathrm{kg}$ respectively), and then AAV vectors were delivered subretinally via a trans-scleral transchoroidal approach $(12,13)$.

Pigs. Eleven-week-old Large White (LW) female piglets were used. Pigs were fasted overnight leaving water ad libitum. The anesthetic and surgical procedures for pigs were previously described (12). Each viral vector was injected in a total volume of $100 \mu$ resulting in the formation of a subretinal bleb with a typical dome-shaped retinal detachment, with a size corresponding to 5 optical discs $(12,13)$.

\section{Human retina}

In collaboration with the Eye Bank of Venice, we collected retina samples from a donor in compliance with the tenets of the Declaration of Helsinki and after obtaining written informed consent from the donor's next of kin.

\section{Subcloning and protein purification}

DNA fragments encoding the sequence of the engineered transcription factors $Z F 6-D B$ and $h K L F 15$, to be expressed as maltose-binding protein (MBP) fusions, were generated by PCR using the plasmids pAAV2.1 CMV-hKLF15 and pAAV2.1 CMV-ZF6-DB as a DNA template. The following oligonucleotides were used as primers: primer 1 (GGAATTCCATATGGTGGACCACTTACTTCCAG) and primer 2 (CGGGATCCTCAGTTCACGGAGCGCACGGAG) for $h K L F 15$; primer 3 (GGAATTCCATATGCTGGAACCTGGCGAAAAACCG) and primer 4 (CGGGATCCCTATCTAGAAGTCTTTTTACCGGTATG) for ZF6-DB. All PCR products were digested with the restriction enzymes NdeI and BamH1 and subcloned into an NdeI- and BamH1-digested pMal C5G (New England Biolabs) bacterial expression vector. All the plasmids obtained were sequenced to confirm that there were no mutations in the coding sequences. The fusion proteins were expressed in the Escherichia coli BL21DE3 host strain. The transformed cells were grown in rich medium plus $0.2 \%$ glucose (according to the protocol from New England Biolabs) at $37^{\circ} \mathrm{C}$ until the absorbance at $600 \mathrm{~nm}$ was $0.6-0.8$, at which time the medium was supplemented with $200 \mu \mathrm{M} \mathrm{ZnSO}_{4}$, and protein expression was induced with $0.3 \mathrm{mM}$ isopropyl 1-thio- $\beta$-D-galactopyranoside and was allowed to proceed for 2 hours. The cells were then harvested, resuspended in $1 \times$ PBS (pH 7.4) containing $1 \mathrm{mM}$ phenylmethylsulfonyl fluoride, $1 \mu \mathrm{M}$ leupeptin, $1 \mu \mathrm{M}$ aprotinin, and $10 \mu \mathrm{g} / \mathrm{ml}$ lysozyme, 
sonicated, and centrifuged for 30 minutes at 90,467 $\mathrm{g}$. The supernatant was then loaded on amylose resin (New England Biolabs) according to the manufacturer's protocol. To remove the MBP from the proteins, bound fusion proteins were cleaved in situ on the amylose resin with factor Xa (1 unit/20 $\mu \mathrm{g}$ of MBP fusion protein) in factor Xa buffer ( $20 \mathrm{mM}$ Tris, $\mathrm{pH} 8.0,100 \mathrm{mM} \mathrm{NaCl}, 2 \mathrm{mM} \mathrm{CaCl}{ }_{2}$ ) for $24-48$ hours at $4^{\circ} \mathrm{C}$ and collected in the same buffer after centrifugation at $500 \mathrm{rpm}$ for 5 minutes. The supernatant containing the protein without the MBP tag was then recovered.

Gel mobility shift analysis

The affinity binding constant of proteins for the $h R H O$ proximal promoter sequence was measured by a gel mobility shift assay by performing a titration of the proteins with the oligonucleotides. The purified proteins were incubated for 15 minutes on ice with an hRHO 65-bp duplex oligonucleotide in the presence of $25 \mathrm{mM}$ Hepes ( $\mathrm{pH} 7.9$ ), $50 \mathrm{mM} \mathrm{KCl}, 6.25 \mathrm{mM} \mathrm{MgCl}_{2}, 1 \%$ Nonidet P-40, and 5\% glycerol. After incubation, the mixture was loaded on a 5\% polyacrylamide gel (29:1 acrylamide/bisacrylamide ratio) and run in $0.5 \mathrm{TBE}$ at $4^{\circ} \mathrm{C}$ ( $200 \mathrm{~V}$ for 4 hours). Protein concentration was determined by a modified version of the Bradford procedure. After electrophoresis, the gel was stained with the fluorescent dye SYBR Green I Nucleic acid gel stain (Invitrogen) to visualize DNA. The hKLF15 protein $(2.5 \mu \mathrm{M})$ was incubated with increasing concentrations $(145,150,170,175,190,195,200,220,240$, and $250 \mathrm{nM})$ of the duplex hRHO 65-bp oligonucleotide. In the case of ZF6-DB, $1.5 \mu \mathrm{M}$ protein was incubated with increasing concentrations $(145,150,170,175,195,210,220,225,240$, and $250 \mathrm{nM})$ of the duplex hRho 65-bp oligonucleotide. Scatchard analysis of the gel shift binding data was performed to obtain the $K_{\mathrm{d}}$ values (12). All numerical values were obtained by computer quantification of the image using a Typhoon FLA 9500 biomolecular imager (GE Healthcare Life Sciences).

\section{qPCR}

RNA from tissues was isolated using an RNAeasy Mini Kit (Qiagen), according to the manufacturer's protocol. cDNA was amplified from $1 \mu \mathrm{g}$ isolated RNA using a QuantiTect Reverse Transcription Kit (Qiagen), as indicated in the manufacturer's instructions.

PCR using the cDNA as template was performed in a total volume of $20 \mu 1$, using $10 \mu 1$ LightCycler 480 SYBR Green I Master Mix (Roche) and $400 \mathrm{nM}$ primers under the following conditions: preincubation, $50^{\circ} \mathrm{C}$ for 5 minutes; cycling, 45 cycles of $95^{\circ} \mathrm{C}$ for 10 seconds, $60^{\circ} \mathrm{C}$ for 20 seconds, and $72^{\circ} \mathrm{C}$ for 20 seconds. Each sample was analyzed in duplicate in 2 independent experiments. Transcript levels of pig retinae were measured by qPCR using the LightCycler 480 (Roche) and the following primers: pRho_forward (ATCAACTTCCTCACGCTCTAC) and pRho_reverse (ATGAAGAGGTCAGCCACTGCC), pGnat1_forward (TGTGGAAGGACTCGGGTATC) and pGnat1_reverse (GTCTTGACACGTGAGCGTA), pArr3_forward (TGACAACTGCGAGAAACAGG) and pArr3_reverse (CACAGGACACCATCAGGTTG), pCrx_forward (GAGCTGGAGTCCTTGTTTGC) and pCrx_reverse (CGTGGAGGATCTTGGAGAAG), pNrl_forward (CAGAGCTGCTGCAGTGTCA) and pNrl_reverse (GTTCAACTCGCGCACAGAC), pKlf15_forward (GCAGGACAGCATCTTGGACT) and pKlf15_ reverse (ACAGGAGCTGGTGTTTTTCG). All of the reactions were standardized against porcine Act $\beta$ using the following primers: Act_Forward (ACGGCATCGTCACCAACTG) and Act_reverse (CTGGGTCATCTTCTCACGG). Transcript levels of mouse retinae were measured by qPCR using the LightCycler 480 and the following primers: mRho_Forward (GACTCTGCCAGCTTTCTTTGCT) and mRho_ Reverse (GCGTCGTCATCTCCCAGTGGA), hRho_Forward (CCATCCCAGCGTTCTTTGCC) and hRho_Reverse (CCTCATCGTCACCCAGTGGG), mGnat1_Forward (GACCGAGCCTCAGAATACCA) and mGnat1_Reverse (GGAGAATTGAGTCTCGATAATACCA). All of the reactions were standardized against murine Act $\beta$ and Gapdh using the following primers: mAct_Forward (CAAGATCATTGCTCCTCCTGA) and mAct_reverse (CATGCTACTCCTGCTTGCTGA), mGapdh_forward (GTCGGTGTGAACGGATTTG) and mGapdh_reverse (CAATGAAGGGGTCGTTGATG).

\section{Immunostaining}

Frozen retinal sections were washed once with PBS and then fixed for 10 minutes in $4 \%$ paraformaldehyde. Sections were blocked and permeabilized with $0.3 \%$ Triton X-100 and 5\% donkey serum in TBS for 1 hour. The primary antibody mouse anti-KLF15 (1:200, Abcam, ab185958) was diluted in a blocking solution and incubated overnight at room temperature. The secondary antibody (Alexa Fluor 594, anti-rabbit 1:1,000; 
Invitrogen) was incubated for 1 hour. Vectashield (Vector Lab Inc.) was used to visualize nuclei. Frozen retinal sections were permeabilized with $0.2 \%$ Triton X-100 and 1\% normal goat serum (NGS) for 1 hour, rinsed in PBS, blocked in 10\% NGS, and then incubated overnight at $4^{\circ} \mathrm{C}$ with rabbit anti-human cone arrestin (hCAR) antibody, provided by Cheryl M. Craft (Doheny Eye Institute, Los Angeles, California, USA) diluted 1:10,000 in 10\% NGS. After 3 rinses with $0.1 \mathrm{M} \mathrm{PBS}$, sections were incubated in goat antirabbit IgG conjugated with Texas Red (Alexa Fluor 594, anti-rabbit 1:1,000; Invitrogen) for 1 hour followed by 3 rinses with PBS. Frozen retinal sections were permeabilized with $0.1 \%$ Triton X-100, rinsed in PBS, blocked in $10 \%$ NGS, and then incubated overnight at $4^{\circ} \mathrm{C}$ in mouse anti-rhodopsin antibody (clone 1D4, Abcam) diluted 1:500 in 10\% NGS. After 3 rinses with $0.1 \mathrm{M}$ PBS, sections were incubated in goat anti-mouse IgG conjugated with Texas Red (Alexa Fluor 594, anti-mouse 1:1,000; Invitrogen) for 1 hour followed by 3 washes with PBS. Frozen retinal sections were permeabilized with $0.1 \%$ Triton X-100, rinsed in PBS, blocked in $10 \%$ NGS, and then incubated overnight at $4^{\circ} \mathrm{C}$ in rabbit $\mathrm{G}_{\alpha+1}(\mathrm{~K}-20)(1: 300$, Santa Cruz Biotechnology, sc-389) in blocking solution. After 3 rinses with $0.1 \mathrm{M}$ PBS, sections were incubated in goat anti-mouse IgG conjugated with Texas Red (Alexa Fluor 594, anti-rabbit 1:500, Invitrogen) for 1 hour followed by 3 washes with PBS.

Mouse eyes were enucleated and fixed with $4 \%$ formaldehyde in $0.1 \mathrm{M}$ sodium phosphate buffer, $\mathrm{pH}$ 7.4 for 16 hours at $4^{\circ} \mathrm{C}$. The tissues were then dehydrated through a graded sucrose series and embedded in OCT. Sections (12 $\mu \mathrm{m}$ thick) were cut. Hematoxylin and eosin (H\&E) staining was performed. Sections were photographed using either a Zeiss 800 confocal microscope (Carl Zeiss) or a Leica Fluorescence Microscope System (Leica Microsystems $\mathrm{GmbH}$ ).

\section{Western blot analyses}

Western blot analysis was performed on harvested retinae. Samples were lysed in hypotonic buffer (10 $\mathrm{mM}$ Tris- $\mathrm{HCl}$ [pH 7.5], $10 \mathrm{mM} \mathrm{NaCl}, 1.5 \mathrm{mM} \mathrm{MgCl}$, 1\% CHAPS, $1 \mathrm{mM}$ PMSF, and protease inhibitors) and $20 \mu \mathrm{g}$ of these lysates was separated by 12\% SDS-PAGE. After the blots were obtained, specific proteins were labeled with anti-rhodopsin (1:1,000; Abcam, 1D4), and anti- $\beta$-tubulin $(1: 10,000$; Sigma-Aldrich) antibodies.

\section{ChIP experiments}

For ChIP experiments, HEK293 cells were transfected by $\mathrm{CaCl}_{2}$ with pAAV2.1 CMV-hKLF15 or pAAV2.1 CMV-eGFP. The cells were harvested after 48 hours. ChIP was performed as follows: cells were homogenized mechanically and cross linked using 1\% formaldehyde in PBS at room temperature for 10 minutes, then quenched by adding glycine at a final concentration of $125 \mathrm{mM}$ and incubated at room temperature for 5 minutes. Cells were washed 3 times in cold $1 \times \mathrm{PBS}$ and then lysed in cell lysis buffer $(5 \mathrm{mM}$ Pipes pH 8.0, $0.5 \%$ IGEPAL, $85 \mathrm{mM} \mathrm{KCl}$ ) for 15 minutes. Nuclei were lysed in nucleus lysis buffer ( $50 \mathrm{mM}$ Tris $\mathrm{HCl} \mathrm{pH}$ 8.0, $10 \mathrm{mM}$ EDTA, 0.8\% SDS) for 30 minutes. Chromatin was sheared using a Covaris s220. The sheared chromatin was immunoprecipitated overnight with anti-KLF15 (clone 2G8) ChIP grade (Abcam, ab81604). The immunoprecipitated chromatin was incubated 3 hours with magnetic protein A/G beads (Invitrogen). Beads were than washed with wash buffer and DNA eluted in elution buffer (50 mM Tris $\mathrm{HCl} \mathrm{pH} \mathrm{8.0,1}$ mM EDTA, 1\% SDS). Real-time PCR was performed using primers for the rhodopsin TSS, hRHOTSSFw (TGACCTCAGGCTTCCTCCTA) and hRHOTSSRv (ATCAGCATCTGGGAGATTGG).

\section{FACS rods sorting}

Porcine retinas transduced with AAV8-hGNAT1-eGFP (dose $1 \times 10^{12} \mathrm{gc}$ ) were disaggregated using a Papain Dissociation System (Worthington Biochemical Corporation) following the manufacturer's protocol. Dissociated retinal cells were analyzed using a BD FACSAria III and sorted, separating eGFP-positive cells (rods) from the eGFP-negative fraction.

\section{Electrophysiological testing}

The method used was described previously $(12,13)$. Briefly, mice were dark-reared for 3 hours and anesthetized. Flash ERGs were evoked by 10-ms light flashes generated through a Ganzfeld stimulator (Costruzione Strumenti Oftalmici) and registered as previously described. ERGs and b-wave thresholds were assessed using the following protocol. Eyes were stimulated with light flashes increasing from -5.2 to $+1.3 \log \mathrm{cd} \cdot \mathrm{s} / \mathrm{m}^{2}$ (which correspond to $1 \times 10^{-5.2}$ to $20.0 \mathrm{~cd} \cdot \mathrm{s} / \mathrm{m}^{2}$ ) in scotopic conditions. The $\log$ unit 
interval between stimuli was $0.3 \log$ from -5.4 to $0.0 \log \mathrm{cd} \cdot \mathrm{s} / \mathrm{m}^{2}$ and $0.6 \log$ from 0.0 to $+1.3 \log \mathrm{cd} \cdot \mathrm{s} / \mathrm{m}^{2}$. For ERG analysis in scotopic conditions the responses evoked by $11 \mathrm{stimuli}$ (from -4 to $+1.3 \log \mathrm{cd} \cdot \mathrm{s} / \mathrm{m}^{2}$ ) with an interval of $0.6 \mathrm{log}$ unit were considered. To minimize the noise, $3 \mathrm{ERG}$ responses were averaged at each $0.6 \log$ unit stimulus from -4 to $0.0 \log \mathrm{cd} \cdot \mathrm{s} / \mathrm{m}^{2}$, while one ERG response was considered for higher $\left(0.0\right.$ to $\left.+1.3 \log \mathrm{cd} \cdot \mathrm{s} / \mathrm{m}^{2}\right)$ stimuli. The time interval between stimuli was 10 seconds from -5.4 to 0.7 $\log \mathrm{cd} \cdot \mathrm{s} / \mathrm{m}^{2}, 30$ seconds from 0.7 to $+1 \log \mathrm{cd} \cdot \mathrm{s} / \mathrm{m}^{2}$, or 120 seconds from +1 to $+1.3 \log \mathrm{cd} \cdot \mathrm{s} / \mathrm{m}^{2}$. a- and $\mathrm{b}$-wave amplitudes recorded in scotopic conditions were plotted as a function of increasing light intensity (from -4 to $+1.3 \log \mathrm{cd} \cdot \mathrm{s} / \mathrm{m}^{2}$ ). The photopic ERG was recorded after the scotopic session by stimulating the eye with ten $10-\mathrm{ms}$ flashes of $20.0 \mathrm{~cd} \cdot \mathrm{s} / \mathrm{m}^{2}$ over a constant background illumination of $50 \mathrm{~cd} / \mathrm{m}^{2}$.

RNA-Seq library preparation, sequencing, and alignment

The 16 libraries were prepared using the TruSeq RNA v2 Kit (Illumina) according to the manufacturer's protocol. Libraries were sequenced on the Illumina HiSeq 1000 platform and in 100-nt paired-end format to obtain approximately 30 million read pairs per sample as reported previously $(12,13)$.

\section{Differential expression analysis}

The data set was composed of 16 samples and 25,325 genes, divided into 3 experimental groups: 6 controls, 4 KLF15-treated, and 6 ZF6-DB-treated $(12,13)$.

\section{Data management}

All analyses, except for the reads quality filtering, alignment, and expression estimates, were performed in the R statistical environment (v.3.2.0) (32). Plots were generated with ggplot2 R/Bioconductor package (v.1.0.1) $(12,13)$.

\section{Statistics}

Data are presented as the mean \pm SEM. Statistical significance was computed using the Student's 2-sided $t$ test and $P$ values less than 0.05 were considered significant. No statistical methods were used to estimate the sample size and no animals were excluded.

\section{Study approval}

Animals. All animal experimentation was performed in accordance with institutional guidelines for animal research and all of the animal studies were approved by the authors. The protocol was approved by the Italian Ministry for Health (IACUC protocol number 114/2015-PR).

Human retinae. The "Fondazione Banca degli Occhi del Veneto" (Eye Bank of Venice) provided retina samples from a donor in compliance with the tenets of the Declaration of Helsinki and after obtaining the written informed consent from the donor's next of kin.

\section{Author contributions}

SB, EMS, NDP, and EM designed experiments and analyzed data. EM performed ERG recordings. MS performed histological analysis. MR performed protein affinity measurements. FC analyzed the RNA-Seq data. CW help draft the manuscript. MLB and DV supervised the in vivo studies in large animals. CG and SR performed subretinal injections in the large animals. FS supervised the subretinal injections in large animal studies and provided reagents. EMS wrote the manuscript and supervised the study.

\section{Acknowledgments}

We thank Fondazione Telethon and the TIGEM (Telethon Institute of Genetics and Medicine) Bioinformatics Core and the TIGEM Vector Core for vector production. We thank Sandro Banfi and Fondazione Banca degli Occhi del Veneto for providing the human retinal sections. We thank Sandro Banfi, Antonella De Matteis, Alberto Auricchio, Davide Cacchiarelli, and Alison Forrester for helpful discussions. This work was supported by the European Research Council/ERC grant 311682 "Allelechoker" (to EMS).

Address correspondence to: Enrico Maria Surace, Via Campi Flegrei 34, 80078 Pozzuoli, Italy. Phone: 39.08.11.923.0634; Email: surace@tigem.it. 
1. Swaroop A, Kim D, Forrest D. Transcriptional regulation of photoreceptor development and homeostasis in the mammalian retina. Nat Rev Neurosci. 2010;11(8):563-576.

2. Levo M, Segal E. In pursuit of design principles of regulatory sequences. Nat Rev Genet. 2014;15(7):453-468.

3. Rohs R, Jin X, West SM, Joshi R, Honig B, Mann RS. Origins of specificity in protein-DNA recognition. Annu Rev Biochem. 2010;79:233-269.

4. Seeman NC, Rosenberg JM, Rich A. Sequence-specific recognition of double helical nucleic acids by proteins. Proc Natl Acad Sci USA. 1976;73(3):804-808.

5. Klug A. The discovery of zinc fingers and their applications in gene regulation and genome manipulation. Annu Rev Biochem. 2010;79:213-231.

6. Pavletich NP, Pabo CO. Zinc finger-DNA recognition: crystal structure of a Zif268-DNA complex at 2.1 A. Science. 1991;252(5007):809-817.

7. Weirauch MT, et al. Evaluation of methods for modeling transcription factor sequence specificity. Nat Biotechnol. 2013;31(2):126-134.

8. Jolma A, et al. DNA-dependent formation of transcription factor pairs alters their binding specificity. Nature. 2015;527(7578):384-388.

9. Reiter F, Wienerroither S, Stark A. Combinatorial function of transcription factors and cofactors. Curr Opin Genet Dev. 2017;43:73-81.

10. Thurman RE, et al. The accessible chromatin landscape of the human genome. Nature. 2012;489(7414):75-82.

11. Hartong DT, Berson EL, Dryja TP. Retinitis pigmentosa. Lancet. 2006;368(9549):1795-1809.

12. Botta S, et al. Rhodopsin targeted transcriptional silencing by DNA-binding. Elife. 2016;5:e12242.

13. Mussolino C, et al. Zinc-finger-based transcriptional repression of rhodopsin in a model of dominant retinitis pigmentosa. EMBO Mol Med. 2011;3(3):118-128.

14. Mo A, et al. Epigenomic landscapes of retinal rods and cones. Elife. 2016;5:e11613.

15. Wingender E, Dietze P, Karas H, Knüppel R. TRANSFAC: a database on transcription factors and their DNA binding sites. Nucleic Acids Res. 1996;24(1):238-241.

16. Pearson R, Fleetwood J, Eaton S, Crossley M, Bao S. Krüppel-like transcription factors: a functional family. Int J Biochem Cell Biol. 2008;40(10):1996-2001.

17. Otteson DC, et al. Kruppel-like factor 15, a zinc-finger transcriptional regulator, represses the rhodopsin and interphotoreceptor retinoid-binding protein promoters. Invest Ophthalmol Vis Sci. 2004;45(8):2522-2530.

18. Gray S, et al. Regulation of gluconeogenesis by Krüppel-like factor 15. Cell Metab. 2007;5(4):305-312.

19. Jeyaraj D, et al. Circadian rhythms govern cardiac repolarization and arrhythmogenesis. Nature. 2012;483(7387):96-99.

20. Lu Y, et al. Kruppel-like factor 15 is critical for vascular inflammation. J Clin Invest. 2013;123(10):4232-4241.

21. Fisch S, et al. Kruppel-like factor 15 is a regulator of cardiomyocyte hypertrophy. Proc Natl Acad Sci USA. 2007;104(17):70747079 .

22. Sasse SK, et al. The glucocorticoid receptor and KLF15 regulate gene expression dynamics and integrate signals through feedforward circuitry. Mol Cell Biol. 2013;33(11):2104-2115.

23. Li T, Snyder WK, Olsson JE, Dryja TP. Transgenic mice carrying the dominant rhodopsin mutation P347S: evidence for defective vectorial transport of rhodopsin to the outer segments. Proc Natl Acad Sci USA. 1996;93(24):14176-14181.

24. White MA, Myers CA, Corbo JC, Cohen BA. Massively parallel in vivo enhancer assay reveals that highly local features determine the cis-regulatory function of ChIP-seq peaks. Proc Natl Acad Sci USA. 2013;110(29):11952-11957.

25. Montana CL, et al. Transcriptional regulation of neural retina leucine zipper (Nr1), a photoreceptor cell fate determinant. $J$ Biol Chem. 2011;286(42):36921-36931.

26. Yu W, et al. Nrl knockdown by AAV-delivered CRISPR/Cas9 prevents retinal degeneration in mice. Nat Commun. $2017 ; 8: 14716$

27. Imbeault M, Helleboid PY, Trono D. KRAB zinc-finger proteins contribute to the evolution of gene regulatory networks. Nature. 2017;543(7646):550-554.

28. Nowick K, Hamilton AT, Zhang H, Stubbs L. Rapid sequence and expression divergence suggest selection for novel function in primate-specific KRAB-ZNF genes. Mol Biol Evol. 2010;27(11):2606-2617.

29. GTEx Consortium, et al. Genetic effects on gene expression across human tissues. Nature. 2017;550(7675):204-213.

30. Auricchio A, Smith AJ, Ali RR. The future looks brighter after 25 years of retinal gene therapy. Hum Gene Ther. 2017;28(11):982-987.

31. Bennett J. Taking stock of retinal gene therapy: looking back and moving forward. Mol Ther. 2017;25(5):1076-1094.

32. Huber W, et al. Orchestrating high-throughput genomic analysis with Bioconductor. Nat Methods. 2015;12(2):115-121

33. Solovei I, et al. Nuclear architecture of rod photoreceptor cells adapts to vision in mammalian evolution. Cell. 2009;137(2):356-368. 